\title{
ESTUDO DA SEPARAÇÃO DO PAR ZIRCÔNIO E HÁFNIO POR TROCA IÔNICA
}

\author{
E.C.B. FELIPE ${ }^{1}$ e A.C.Q. LADEIRA ${ }^{2}$ \\ Centro de Desenvolvimento da Tecnologia Nuclear, CDTN/CNEN, Belo Horizonte, MG, Brasil \\ elainecfelipe@yahoo.com.br ${ }^{1}$ - ana.ladeira@cdtn.br ${ }^{2}$
}

Artigo submetido em novembro/2013 e aceito em dezembro/2013

DOI: http://dx.doi.org/10.15628/holos.2014.1769

\section{RESUMO}

O zircônio e o háfnio são dois importantes metais para a indústria nuclear. O háfnio ocorrer em todos os minérios de zircônio na faixa de 2 - 3\%. Entretanto, o uso do zircônio na indústria nuclear exige que o háfnio esteja em concentrações menores que $100 \mathrm{mg} \mathrm{Kg}{ }^{-1}$. O atual trabalho consiste na separação do par zircônio e háfnio pelo método de troca iônica a fim de se obter um concentrado de zircônio de alta pureza. Os licores de zircônio e háfnio foram produzidos a partir da lixiviação de seus hidróxidos $\mathrm{Zr}(\mathrm{OH})_{4}$ e $\mathrm{Hf}(\mathrm{OH})_{4}$ em meio nítrico por 24 horas. A partir destes dois licores foi preparada uma solução contendo $7,5 \times 10^{-2} \mathrm{~mol} \mathrm{~L}^{-1}$ de $\mathrm{Zr}$ e $5,8 \times 10^{-3} \mathrm{~mol} \mathrm{~L}^{-1}$ de $\mathrm{Hf}$, acidez de $1 \mathrm{M}$. Os experimentos de troca iônica foram realizados em batelada, com as resinas Dowex 50WX4 50, Dowex 50WX8 100, Dowex 50WX8 50, Amberlite IR-120 e Marathon C a temperatura constante de $28^{\circ} \mathrm{C}$. As demais variáveis tais como acidez e agitação foram mantidas constantes. A partir do ajuste dos dados a equação de Langmuir foram calculados o carregamento máximo $\left(q_{\max }\right)$, o coeficiente de distribuição $(\mathrm{Kd})$ para o $\mathrm{Zr}$ e $\mathrm{Hf}$ e o fator de separação $\left(\alpha_{\mathrm{Hf}}^{\mathrm{Zr}}\right)$. Os resultados de $\mathrm{q}_{\max }$ de $\mathrm{Zr}$ e $\mathrm{Hf}$, em mmol $\mathrm{g}^{-1}$, mostraram que as resinas mais apropriadas para a remoção em colunas são: Dowex $50 \mathrm{WX} 450$ ( $q_{\max } \mathrm{Zr}=2,21, \mathrm{Hf}=0,18$ ), Dowex 50WX8 50 ( $q_{\max } Z r=1,89, \mathrm{Hf}=0,13$ ) e Amberlite $\left(q_{\max } Z r=1,64, \mathrm{Hf}=\right.$ $0,12)$. A seletividade das resinas para $\mathrm{Zr}$ e Hf foi estudada a partir dos fatores de separação que demonstraram que as resinas praticamente não apresentam seletividade. Ensaios de eluição seletiva serão conduzidos visando a separação do par iônico.

PALAVRAS-CHAVE: zircônio, háfnio, troca iônica.

\section{SEPARATION OF ZIRCONIUM FROM HAFNIUM BY ION EXCHANGE}

\begin{abstract}
The current work consists in the separation of zirconium and hafnium by the ion exchange method in order to obtain a zirconium concentrate of high purity. The zirconium and hafnium liquors were produced from the leaching of the $\mathrm{Zr}(\mathrm{OH})_{4}$ and $\mathrm{Hf}(\mathrm{OH})_{4}$ with nitric acid for 24 hours. From these two liquors it was prepared one solution containing $7,5 \times 10^{-2} \mathrm{~mol} \mathrm{~L}^{-1}$ of $\mathrm{Zr}$ and $5,8 \times 10^{-3} \mathrm{~mol}$ $\mathrm{L}^{-1}$ of $\mathrm{Hf}$ with acidity of $1 \mathrm{M}$. Ion exchange experiments were carried out in batch with the resins Dowex 50WX4, Dowex 50WX8 100, Dowex 50WX8 50, Amberlite IR-120 and Marathon $\mathrm{C}$ at constant temperature $28^{\circ} \mathrm{C}$. Other
\end{abstract}

variables such as, acidity and agitation were kept constant. The data were adjusted to Langmuir equation in order to calculate the maximum loading capacity ( $q_{\max }$ ), the distribution coefficient $(\mathrm{Kd})$ for $\mathrm{Zr}$ and $\mathrm{Hf}$ and the separation factor $\left(\alpha_{\mathrm{Hf}}^{\mathrm{Zr}}\right)$. The results of maximum loading capacity $\left(q_{\max }\right.$ ) for $\mathrm{Zr}$ and $\mathrm{Hf}$, in $\mathrm{mmol} \mathrm{g}^{-1}$, showed that the most suitable resins for columns experiments are: Dowex 50WX4 50, Dowex 50WX8 50 and Amberlite. However, separations factors showed that the resins are not selective.

KEYWORDS: zirconium, hafnium, ion exchange. 


\section{INTRODUÇÃO}

O Zircônio é um metal de grande valor para indústria nuclear. Suas ligas se destacam na aplicação como material de revestimento de pastilhas combustíveis e em reatores nucleares. $\mathrm{Na}$ indústria química o zircônio é utilizado em ligas com níquel para obtenção de materiais resistentes à corrosão. O óxido de zircônio impuro é empregado na fabricação de tijolos para fornos de alumínio na indústria de cerâmica. Na indústria eletrônica o zircônio é utilizado em placas e filamentos. Além disto, as ligas de zircônio e nióbio podem ser utilizadas na fabricação de imãs supercondutores por apresentarem supercondutividade em baixas temperaturas.

O háfnio ocorre em todos os minérios de zircônio na faixa de 2 - 3\%, mas o uso do zircônio na indústria nuclear exige que este tenha concentrações menores que $100 \mathrm{mg} \mathrm{kg}^{-1}$ de háfnio (pureza nuclear). A separação do par $\mathrm{Zr} / \mathrm{Hf}$ é difícil devido à similaridade de suas propriedades químicas como, raio atômico, raio iônico e eletronegatividade (SMOLIK et al., 2009). O háfnio por sua vez, também é utilizado na indústria nuclear como material constituinte de barras de controle em reatores nucleares, devido a sua alta capacidade de absorção de nêutrons térmicos. Entretanto, a principal aplicação do háfnio é como aditivo (1 - 2\%) em superligas a base de níquel para a fabricação das hélices das turbinas de aeronaves (HABASHI, 1997).

Os sais de o zircônio e háfnio podem apresentar valência $4^{+}, 3^{+}, 2^{+}$ou $1^{+}$, porém, em solução aquosa o estado de oxidação mais comum é $4^{+}$. Entretanto, outras espécies aniônicas ou catiônicas podem ser formadas dependo da acidez do meio (BENEDICT et al., 1953; D'OLIESLAGER e COMEYNE 1987; FIATY et al., 2007; HABASHI, 1997; PORIEL et al., 2006). Connick e McVey (1948) relatam que o zircônio e háfnio em soluções muito ácidas não sofrem hidrolise, estando preferencialmente na forma tetravalente.

Numerosos métodos têm sido utilizados para separar este par iônico. Tais métodos incluem cristalização fracionária, troca iônica, destilação fracionada, difusão térmica, extração por solventes e separação eletroquímica (OVERHOLSER et al., 1960). O presente trabalho consiste na separação do par zircônio e háfnio pelo método de troca iônica a fim de se obter um concentrado de zircônio de alta pureza, que deverá ser utilizado para obtenção da esponja de zircônio. A aplicação da troca iônica no processo de separação do par Zr/Hf oferece muitas vantagens, uma vez que permite a recuperação de íons em soluções muito diluídas e a possibilidade de processar grandes volumes de soluções onde operações de precipitação ou extração com solventes seriam desfavoráveis.

\section{METOdOLOGIA}

\subsection{Amostras}

As amostras de hidróxidos de zircônio e háfnio para a produção dos licores foram fornecidas pelo Centro Tecnológico da Marinha de São Paulo (CTM-SP). Os teores de Zr e Hf nas amostras de $\mathrm{Zr}(\mathrm{OH})_{4}$ e $\mathrm{Hf}(\mathrm{OH})_{4}$, após caracterização por espectrometria de fluorescência de raios-X por energia dispersiva (equipamento Shimadzu modelo EDX 720), estão apresentados na tabela 1. 
Tabela 1 - Caracterização dos hidróxidos de zircônio de háfnio.

\begin{tabular}{ccc}
\hline Hidróxidos & Zircônio(\%) & Háfnio(\%) \\
\hline $\mathrm{Zr}(\mathrm{OH})_{4}$ & 76,26 & 0,08 \\
$\mathrm{Hf}(\mathrm{OH})_{4}$ & 11,20 & 57,30 \\
\hline
\end{tabular}

\subsection{Licores}

Os licores, contendo os elementos zircônio e háfnio, foram produzidos a partir da lixiviação de seus respectivos hidróxidos. As condições de lixiviação foram: meio nítrico $7 \mathrm{M}$ para $\mathrm{Zr}(\mathrm{OH})_{4} \mathrm{e}$ $8 \mathrm{M}$ para $\mathrm{Hf}(\mathrm{OH})_{4}$, temperatura ambiente $\left(25 \pm 2^{\circ} \mathrm{C}\right)$ e tempo de contato de 24 horas. $\mathrm{O}$ licor de zircônio produzido pela lixiviação apresentou concentração de $5,8 \times 10^{-1} \mathrm{~mol} \mathrm{~L}^{-1}$ de $\mathrm{Zr}$ e $5,3 \times 10^{-4} \mathrm{~mol}$ $\mathrm{L}^{-1}$ de Hf. O licor de háfnio apresentou $2,9 \times 10^{-1} \mathrm{~mol} \mathrm{~L}^{-1}$ de $\mathrm{Hf}$ e $7,2 \times 10^{-2} \mathrm{~mol} \mathrm{~L}^{-1}$ de $\mathrm{Zr}$.

\subsection{Resinas}

Todas as resinas utilizadas são catiônicas fortes na forma de $\mathrm{H}^{+}$, grupo funcional $-\mathrm{SO}_{3} \mathrm{H}$ (ácido sulfônico). As resinas, Dowex 50WX4 50-100, Dowex 50WX8 100-200, Dowex 50WX8 50100, Amberlite IR-120 e Marathon C, foram selecionadas de acordo com a literatura e orientação dos fabricantes.

\subsection{Isotermas de troca iônica}

A partir dos licores de $\mathrm{Zr}$ e Hf foi preparada uma solução com $7,5 \times 10^{-2} \mathrm{~mol} \mathrm{~L}^{-1}$ de zircônio e $5,8 \times 10^{-3} \mathrm{~mol} \mathrm{~L}^{-1}$ de háfnio com acidez de $1 \mathrm{M}$. Os ensaios para a obtenção das isotermas de troca iônica foram realizados em alíquotas de $100 \mathrm{~mL}$ da solução anterior e massa de resina entre 0,5 a $6,5 \mathrm{~g}$. As misturas foram agitadas a $140 \mathrm{~min}^{-1}$, em um agitador horizontal MA830 da marca Marconi com temperatura controlada em $28^{\circ} \mathrm{C}$ durante 24 horas. Após a agitação, a solução foi filtrada e enviada para analise da concentração dos metais por espectrometria de fluorescência de raios-X por energia dispersiva (equipamento Shimadzu modelo EDX 720). A quantidade de zircônio e háfnio adsorvido pelas resinas foi determinada através da equação (1).

$$
\mathrm{Qeq}=(\mathrm{Ci}-\mathrm{Ceq}) \times \mathrm{V} / \mathrm{m}
$$

Onde Qeq é a concentração do íon na a fase sólida no equilíbrio ( $\mathrm{mmol} \mathrm{g}^{-1}$ ), Ci é a concentração inicial de íon na fase líquida $\left(\mathrm{mmol} \mathrm{L}^{-1}\right)$, Ceq é a concentração do íon na fase líquida no equilíbrio ( $\left.\mathrm{mmol} \mathrm{L}^{-1}\right), \mathrm{V}$ é o volume da solução (L) e $\mathrm{m}$ é a massa de adsorvente $(\mathrm{g})$. A quantidade máxima ( $q_{\max }$ em $\mathrm{mmol} \mathrm{g}^{-1}$ ) de zircônio e háfnio removido pelas diferentes resinas foi calculada a partir do ajuste dos dados a equação (2) de Langmuir (McCABE et al., 1993).

\section{Qeq $=(q \max . \mathrm{b} \cdot$ Ceq $) /(1+$ b.Ceq $)$}

Onde, $q_{\max }$. é a capacidade máxima de troca iônica $\left(m m o l g^{-1}\right)$, e b é a constante de Langmuir.

Os coeficientes de distribuição $\mathrm{K}_{d}\left(\mathrm{~mL} \mathrm{~g}^{-1}\right)$ para o $\mathrm{Zr}$ e o $\mathrm{Hf}$ foram calculados a partir dos dados das isotermas utilizando a equação (3) citada em Poriel et al. (2006), onde o V é o volume da solução $(\mathrm{mL})$. 
O fator de separação foi calculado utilizando a equação (4) e é razão entre os índices de concentração dos íons de $\mathrm{Zr}$ e $\mathrm{Hf}$ na resina $\left(\overline{\mathrm{C}}_{\mathrm{Zr}}, \overline{\mathrm{C}}_{\mathrm{Hf}}\right)$ e na fase líquida $\left(\mathrm{C}_{\mathrm{Zr}}, \mathrm{C}_{\mathrm{Hf}}\right)$ Se o íon $\mathrm{Zr}$ for preferido pelo trocador iônico o $\alpha_{\mathrm{Hf}}^{\mathrm{Zr}}>1$, caso o íon $\mathrm{Hf}$ seja preferido o $\alpha_{\mathrm{Hf}}^{\mathrm{Zr}}<1$ (HELFFERICH, 1995).

$$
\alpha_{\mathbf{H f}}^{\mathrm{Zr}}=\frac{\overline{\mathrm{C}}_{\mathrm{Zr}} \mathrm{C}_{\mathbf{H f}}}{\overline{\mathbf{C}}_{\mathbf{H f}} \mathrm{C}_{\mathbf{Z r}}}
$$

\section{RESULTADOS E DISCUSSÃO}

\subsection{Isotermas de troca iônica}

Isotermas de troca iônica serão utilizadas no presente trabalho para descrever a relação entre a quantidade de zircônio e háfnio adsorvidos na resina e a concentração desses metais na fase líquida em uma determinada temperatura. Os trabalhos de Donia et al. (2011), Fiaty et al. (2007) e Xu et al. (2010) mostram que a utilização da isoterma de Langmuir em estudos de troca iônica envolvendo os íons $\mathrm{Zr}^{4+}$ e $\mathrm{Hf}^{4+}$ tem sido satisfatório. Os dados desta etapa foram ajustados de acordo com a equação de Langmuir (2).

A figura 1 apresenta as isotermas para cada resina assim como o carregamento máximo de zircônio ( $q_{\max }$ ) em mmol g-1.

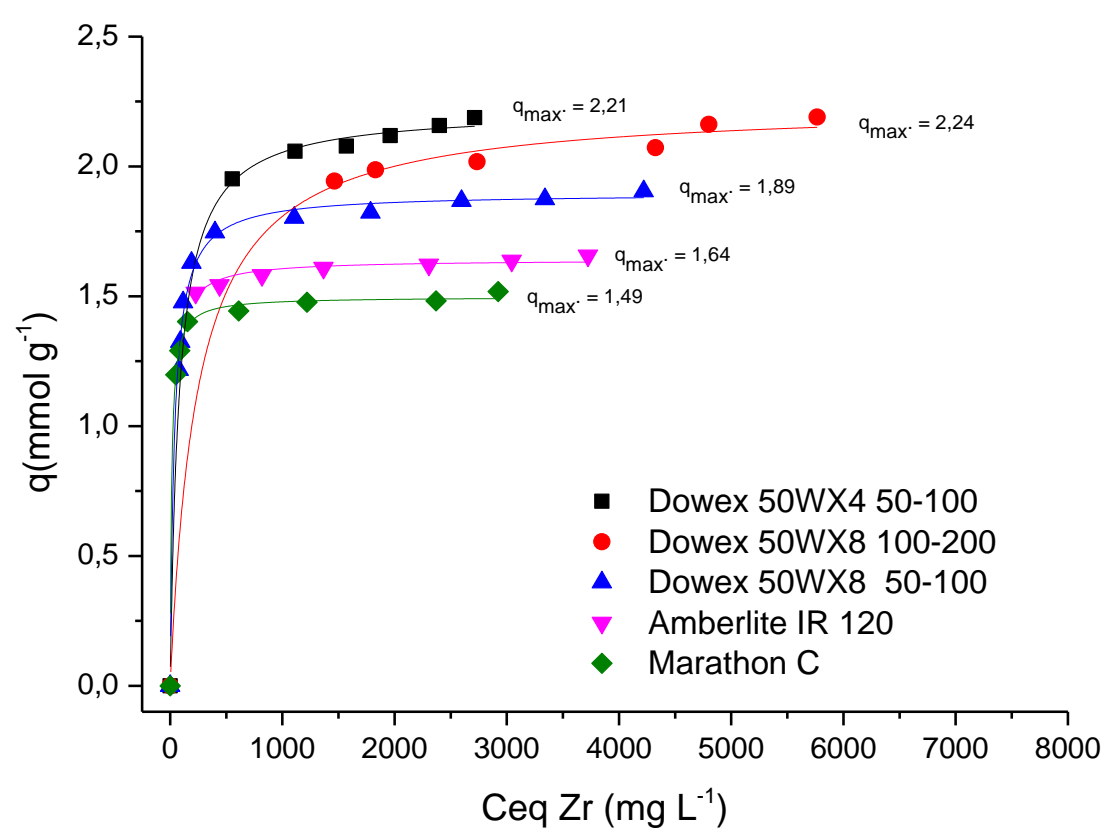

Figura 1: Isoterma de troca iônica para o zircônio. Condições experimentais: $T=28^{\circ} \mathrm{C}$, agitação $=140 \mathrm{~min}^{-1},[\mathrm{Zr}]_{\mathrm{inicial}}$ $=7,2 \times 10^{-2} \mathrm{~mol} \mathrm{~L}^{-1} \mathrm{e}[\mathrm{Hf}]_{\text {inicial }}=5,6 \times 10^{-3} \mathrm{~mol} \mathrm{~L}^{-1}$ para a resina Marathon $\mathrm{C}$ e solução contendo $[\mathrm{Zr}]_{\text {inicial }}=7,4 \times 10^{-2} \mathrm{~mol} \mathrm{~L}^{-}$ ${ }^{1} \mathrm{e}[\mathrm{Hf}]_{\text {inicial }}=5,5 \times 10^{-3} \mathrm{~mol} \mathrm{~L}^{-1}$ para as demais resinas, acidez $1 \mathrm{M} \mathrm{HNO}_{3}$. 
A partir do ajuste dos dados a equação de Langmuir foram calculados os carregamentos máximos $\left(q_{\max }\right)$ para cada resina. De acordo com o a Figura 1, nota-se que os melhores carregamentos para o zircônio $\left(q_{\max z r}\right)$ foram apresentados pelas resinas Dowex 50WX8 100-200 $\left(2,24 \mathrm{mmol} \mathrm{g}^{-1}\right)$, seguida da Dowex 50WX4 50-100 (2,21 mmol g$\left.{ }^{-1}\right)$ e Dowex 50WX8 50-100 (1,89 $\left.\mathrm{mmol} \mathrm{g}^{-1}\right)$. A recuperação de zircônio variou de 50,88 a 71,18 \%. Embora, a resina Dowex $50 \mathrm{WX} 8$ 100-200 tenha apresentado o maior carregamento para o zircônio, esta não será utilizada na próxima etapa de ensaios de troca iônica em coluna de leito fixo, uma vez que a sua menor granulometria tornaria inviável a sua aplicação em escala industrial.

A Figura 2 apresenta as isotermas para cada resina assim como o carregamento máximo de háfnio $\left(q_{\max }\right)$ em mmol g-1.

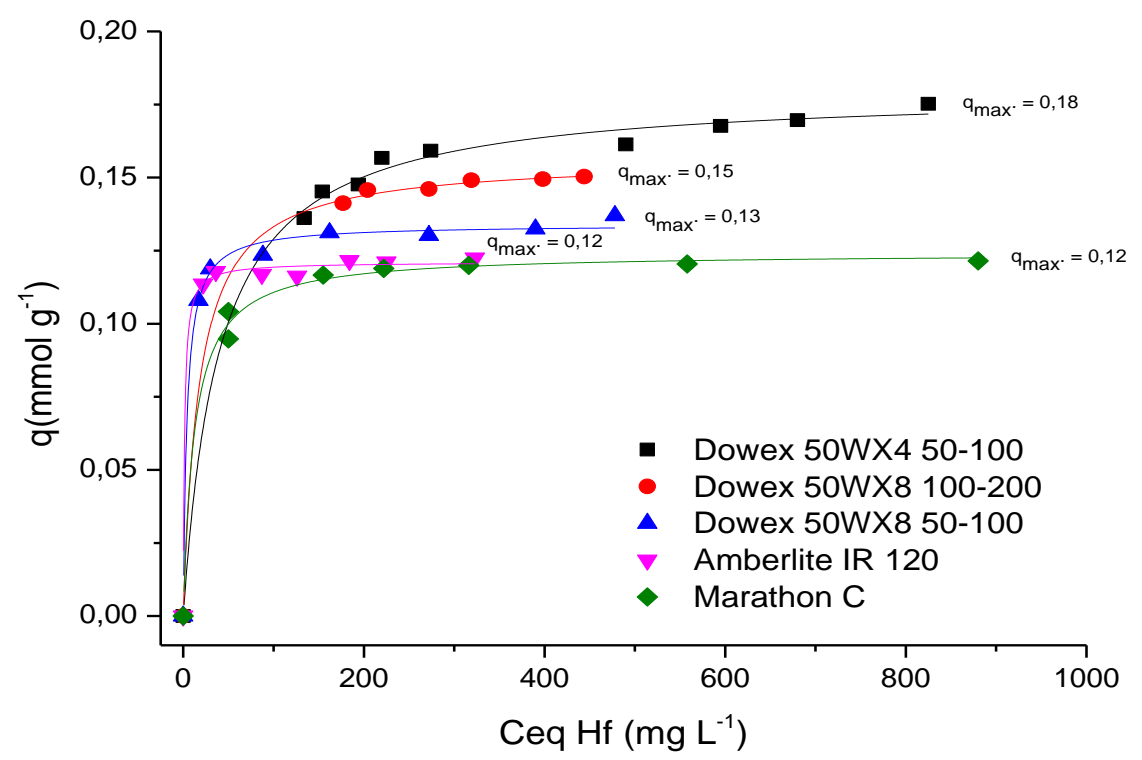

Figura 2: Isoterma de troca iônica para o háfnio. Condições experimentais: $\mathrm{T}=28^{\circ} \mathrm{C}$, agitação $=140 \mathrm{~min}^{-1}$, $[\mathrm{Hf}]_{\text {inicial }}$ $=5,6 \times 10^{-3} \mathrm{~mol} \mathrm{~L}^{-1} \mathrm{e}[\mathrm{Zr}]_{\text {inicial }}=7,2 \times 10^{-2} \mathrm{~mol} \mathrm{~L}^{-1}$ para a Marathon $\mathrm{C}$; e solução contendo $[\mathrm{Hf}]_{\text {inicial }}=5,5 \times 10^{-3} \mathrm{~mol} \mathrm{~L}^{-1} \mathrm{e}$ $[\mathrm{Zr}]_{\text {inicial }}=7,4 \times 10^{-2} \mathrm{~mol} \mathrm{~L}^{-1}$ para as demais resinas; acidez $1 \mathrm{M} \mathrm{HNO}_{3}$.

Conforme a Figura 2, as resinas que apresentaram melhor troca iônica para o háfnio foram Dowex 50WX4 50-100, Dowex 50WX8 100-200 e 50WX8 50-100, seguida das resinas Amberlite IR 120 e Marathon C. Os valores de $q_{\max }$ variaram de 0,12 a $0,18 \mathrm{mmol} \mathrm{g}^{-1}$ e a recuperação variou de 51,08 a $71,34 \%$.

Todas as isotermas de troca iônica do zircônio e háfnio apresentaram um comportamento referente ao tipo de isoterma conhecida como extremamente favorável, onde se observa uma afinidade do íon pelo trocador e a diminuição dos sítios disponíveis para a troca iônica à medida que a concentração do íon na fase aquosa aumenta (MCCABE et al., 1993).

$\mathrm{Na}$ tabela 2 estão apresentados os valores de carregamento máximo do zircônio e háfnio $\left(\mathrm{mmol} \mathrm{g}{ }^{-1}\right.$ ), conforme valores das figuras 1 e 2 , os coeficiente de distribuição $\left(K_{d}\right)$ e fator de separação $\left(\alpha_{\mathrm{Hf}}^{\mathrm{Zr}}\right)$ das resinas utilizadas. 
Tabela 2: Carregamento máximo $\left(q_{\max }\right)$ zircônio e háfnio, coeficiente de distribuição $\left(K_{d}\right)$ e fator de separação do zircônio e háfnio $\left(\alpha_{\mathrm{Hf}}^{\mathrm{Zr}}\right)$ nas resinas testadas.

\begin{tabular}{lccccc}
\hline \multicolumn{1}{c}{ Resina } & $\begin{array}{c}\mathrm{q}_{\text {maxZr }} \\
\mathrm{mmol} \mathrm{g}^{-1}\end{array}$ & $\begin{array}{c}\mathrm{q}_{\text {maxHf }} \\
\mathrm{mmol} \mathrm{g}^{-1}\end{array}$ & $\begin{array}{c}\mathrm{K}_{\mathrm{dZr}} \\
\mathrm{mL} \mathrm{g}^{-1}\end{array}$ & $\begin{array}{c}\mathrm{K}_{\mathrm{dHf}} \\
\mathrm{mL} \mathrm{g}^{-1}\end{array}$ & $\alpha_{H f}^{Z r}$ \\
\hline Dowex 50WX4 50 & 2,21 & 0,18 & 98,61 & 106,0 & 0,93 \\
Dowex 50WX8 100 & 2,24 & 0,15 & 67,52 & 69,32 & 0,98 \\
Dowex 50WX8 50 & 1,89 & 0,13 & 49,75 & 52,83 & 0,98 \\
Marathon C & 1,49 & 0,12 & 57,39 & 67,79 & 0,84 \\
Amberlite IR 120 & 1,64 & 0,12 & 64,40 & 68,61 & 0,98 \\
\hline
\end{tabular}

As resinas testadas apresentaram maior $q_{\text {máx }}\left(\mathrm{mmol} \mathrm{g}^{-1}\right)$ para o zircônio. Acredita-se que estes valores são atribuídos a maior disponibilidade desta espécie no meio, uma vez que, a concentração do zircônio é muito maior que a concentração do háfnio. Xu et al (2010) relatam esse fato ao descrever uma maior capacidade de troca iônica para $\mathrm{Zr}\left(q_{\text {máx. }}=5,19 \mathrm{mmol} \mathrm{g}^{-1}\right)$ em relação ao $\mathrm{Hf}\left(q_{\text {máx. }}=8 \times 10^{-2} \mathrm{mmol} \mathrm{g}^{-1}\right)$ em estudo de separação do $\mathrm{Zr} / \mathrm{Hf}$ em resina de extração MIBK (metilisobutilcetona). Outro estudo, realizado por Donia et al (2011) propõe o emprego de uma resina magnética quelante catiônica para investigar a separação do zircônio e háfnio utilizando-se uma solução com $2,2 \times 10^{-3} \mathrm{~mol} \mathrm{~L}^{-1} \mathrm{Zr}$ e $6,1 \times 10^{-5} \mathrm{~mol} \mathrm{~L}^{-1} \mathrm{Hf}$ e $\mathrm{pH}$ 2.5. Os resultados alcançados para carregamento máximo, conforme modelo de Langmuir, foram: $0,96 \mathrm{mmol} \mathrm{g}^{-1} \mathrm{Zr}$ e $0,01 \mathrm{mmol} \mathrm{g}^{-1}$ $\mathrm{Hf}$. Entretanto, as concentrações iniciais utilizadas no presente trabalho, i.e, $[\mathrm{Zr}]=7 \times 10^{-2} \mathrm{~mol} \mathrm{~L}^{-1} \mathrm{e}$ $[\mathrm{Hf}]=5,8 \times 10^{-3} \mathrm{~mol} \mathrm{~L}^{-1}$, são maiores que as investigadas pelos autores anteriores e favorecem um maior $q_{\max }$. conforme verificado.

Faghihian e Kabiri-Tadi (2010) estudaram a remoção do zircônio de soluções aquosas através do uso de zeolitas nas formas $\mathrm{Na}^{+}, \mathrm{K}^{+}, \mathrm{Ca}^{+}$e na forma natural em ensaios de adsorção com solução $[\mathrm{Zr}]=1 \times 10^{-2} \mathrm{~mol} \mathrm{~L}^{-1}$, temperatura $40 \circ \mathrm{C}$ e pH 1,85. O ajuste dos dados ao modelo de Langmuir apresentou um carregamento máximo de zircônio de $9,1 \times 10^{-2} \mathrm{~mol} \mathrm{~L}^{-1}$ zeolita natural e $6,7 \times 10^{-2} \mathrm{~mol} \mathrm{~L}^{-1}$ zeolita $\mathrm{Na}^{+}$.

A acidez elevada do meio também pode altera o qmax para o metal. Segundo Xu et al. (2010) a troca iônica do $\mathrm{Zr}$ e $\mathrm{Hf}$ diminui com o aumento da acidez do meio $(>2,5 \mathrm{M})$ devido a competição do $\mathrm{H}^{+}$pelo sitio da resina. Portanto, todos os ensaios de troca iônica deste trabalho foram realizados em acidez $1 \mathrm{M}$ favorecendo um maior carregamento $\mathrm{Zr}$ e $\mathrm{Hf}$.

A distribuição dos íons de zircônio e háfnio na resina é indicada através dos seus respectivos coeficientes de distribuição $\left(K_{d}\right)$. Os valores de $K_{d}$ da Tabela 2 indicam que o háfnio distribui levemente melhor do que o zircônio em todas as resinas testadas, confirmando que estas apresentam uma maior afinidade pelo $\mathrm{Hf}$ em relação ao Zr. De forma contrária, Poriel et al. (2006) obtiveram um $\mathrm{K}_{\mathrm{dzr}}=90,4$ e $\mathrm{KdHf}_{\mathrm{dH}}=8,7$ em estudos de separação do $\mathrm{Zr} / \mathrm{Hf}$ utilizando resinas aniônica em solução $[\mathrm{Zr}]=2,1 \times 10^{-2} \mathrm{~mol} \mathrm{~L}^{-1},[\mathrm{Hf}]=8,7 \times 10^{-3} \mathrm{~mol} \mathrm{~L}^{-1}$ e acidez $9,5 \mathrm{M} \mathrm{HCl}$.

A preferência do trocador iônico por um dos íons presentes na solução também pode ser expressa pelo fator de separação $\left(\alpha_{\mathrm{Hf}}^{Z r}\right)$. Se o íon $\mathrm{Zr}$ for preferido pelo trocador iônico o $\alpha_{\mathrm{Hf}}^{\mathrm{Zr}}>1$, caso o íon $\mathrm{Hf}$ seja preferido o $\alpha_{\mathrm{Hf}}^{\mathrm{Zr}}<1$. Porém, apesar da elevada concentração de $\mathrm{Zr}$, as resinas não correspondem à expectativa de uma maior seletividade para o $\mathrm{Zr}$ em relação ao Hf. Este fato pode ser verificado pelos valores próximos de 1 para os fatores de separação $\left(\alpha_{\mathrm{Hf}}^{\mathrm{Zr}}\right)$. Apesar de estarem próximos de 1, todos os $\alpha_{\mathrm{Hf}}^{\mathrm{Zr}}<1$, ou seja, existe uma tendência das resinas em serem mais seletivas para o háfnio. Pode-se afirmar com base na Tabela 2 que a resina que melhor separou o háfnio foi 
a Marathon $\mathrm{C} \alpha_{\mathrm{Hf}}^{\mathrm{Zr}}=0,84$. As demais resinas, embora tenham apresentado $\alpha_{\mathrm{Hf}}^{\mathrm{Zr}}<1$, não se apresentam seletividade uma vez que o fator de separação é muito próximo a 1.

A literatura apresenta poucos dados de fatores de separação em relação à separação do par Zr e Hf. Os estudos de Donia et al. (2011) com uma mistura sintética contendo $[\mathrm{Zr}]=2 \times 10^{-3} \mathrm{~mol}$ $\mathrm{L}^{-1} \mathrm{e}[\mathrm{Hf}]=6 \times 10^{-5} \mathrm{~mol} \mathrm{~L}^{-1}$ e uma amostra real $[\mathrm{Zr}]=4.8 \times 10^{-3} \mathrm{~mol} \mathrm{~L}^{-1} \mathrm{e}[\mathrm{Hf}]=3 \times 10^{-4} \mathrm{~mol} \mathrm{~L}^{-1} \mathrm{em} \mathrm{pH}=2,5$ mostram que a resina magnética catiônica mais seletiva para o $\mathrm{Zr}$ em relação ao háfnio, pois os fatores de separação são $\alpha_{\mathrm{Hf}}^{\mathrm{Zr}}=5$ e $\alpha_{\mathrm{Hf}}^{\mathrm{Zr}}=6$, respectivamente.

A seletividade da resina por um íon aumenta com o aumento da valência, visto que, este íon é fortemente atraído pelo íon fixo da resina. O numero atômico também favorece a seletividade da resina, quanto maior o numero atômico, maior a preferência da resina por este íon (HELFFERICH, 1995). O zircônio e háfnio apresentam valência $4^{+}$, portanto, não haveria preferência por nenhum dos íons, porém, o háfnio possui numero atômico $(z=72)$ maior que o do $\operatorname{Zr}(z=40)$, tornando assim preferido pelas resinas, confirmando os valores de $\alpha_{\mathrm{Hf}}^{\mathrm{Zr}}<1$ para as resinas avaliadas. O aumento das ligações cruzadas também aumenta a seletividade das resinas (HABASHI, 1999). Todas as resinas possuem $8 \%$ de ligação cruzada de divinilbenzeno (DVB), exceto Dowex 50WX4 (4\% DVB). Entretanto, analisando o $\alpha_{\mathrm{Hf}}^{\mathrm{Zr}}$ esta ultima não se apresentou menos seletiva conforme esperado.

\section{CONCLUSÃO}

Os resultados de carregamento máximo obtido através das isotermas de Langmuir demonstraram que as resinas apresentaram maior carregamento para o Zr. Porém, todas as resinas utilizadas apresentaram uma ligeira maior seletividade para o háfnio. Tal fato é evidenciado

pelo $\alpha_{\mathrm{Hf}}^{\mathrm{Zr}}<1$, sendo que a resina Marathon $\mathrm{C} \alpha_{\mathrm{Hf}}^{\mathrm{Zr}}=0,84$ apresentou a melhor separação do háfnio. As resinas Dowex 50WX4, 50WX8 100 e 50WX8 50 não se mostraram seletivas, apesar de serem resinas comerciais indicadas para separação do par $\mathrm{Zr} / \mathrm{Hf}$ em cromatografia. Ensaios em coluna estão em andamento, onde será avaliada a eluição seletiva do $\mathrm{Zr}$ ou do $\mathrm{Hf}$ como uma forma de viabilizar o uso destas resinas na separação do par iônico estudado. Além disso, deverá ser avaliada a definição das variáveis operacionais como vazão, tempo de residência, altura de leito, entre outras.

\section{REFERÊNCIAS BIBLIOGRÁFICAS}

1. BENEDICT, J. T.; SCHUMB, W. C.; CORYELL, C. D. Distribution of zirconium and hafnium between cation-exchange resin and acid solutions. The colunm separation with nitric acidcitric acid mixture. Journal of the American Chemical Society, v. 76, p. 2036-2040, 1953.

2. CONNICK, R. E.; MCVEY, W. H. The aqueous chemistry of zirconium. Journal of the American Chemical Society, p. 3182-3191, 1949.

3. D'OLIESLAGER, W.; COMEYNE, D. Properties of $\mathrm{Zr}(\mathrm{IV})$ and Hf(IV) ions on Dowex 50WX8: An ion exchange equilibrium study. Reactive Polymers, Amsterdam, v. 7, p. 133-139, 1988.

4. DONIA, A.M., ATIA, A.A., DAHER. A. M., ELSHEHY, E.A. Extraction and separation of zirconium(IV) and hafnium(IV) from chloride media using magnetic resin with phosphoric acid functionality. Jouranl of Dispersion Science and Technology., v. 32, p. 193-202, 2011. 
5. FAGHIHIAN, H., KABIRI-TADI, M. Removal of zirconium from aqueous solution by modified clinoptilolite. Journal of Hazardous Materials., v. 178, p. 66-73, 2010.

6. FIATY, K., LAURENT, P., PORIEL, L., PELLET-ROSTAING, S., LEMAIRE, M.. Solid/liquid extraction of zirconium and hafnium in hydrochloric acid aqueous solution with anion exchange resinkinetic study and equilibrium analyses. Industrial and Engineering Chemistry Research, v. 46, n. 4, p. 1286-1291, 2007.

7. HABASHI, F. Handbook of Extractive Metallurgy. WILEY-VCH, v. 3, 1997. 1431-1469 p.

8. HABASHI, F. Textbook of Hydrometallurgy. Second. ed. Quebec: Métallurgiie Extractive Québec, 1999. 406-420 p.

9. HELFFERICH, F. Ion Exchange. New York: Dover Publications, INC., 1995. 35-153 p.

10. McCABE, W. L.; SMITH, J. C.; HARRIOTT, P. Unit Operations of Chemical Engineering. Fifth. ed. McGraw-Hill Chemical Engineering Series, 1993. 810-820 p.

11. OVERHOLSER, L. G.; BARTON, C. J.; RAMSEY, J. W. Separation of hafnium from zirconium. 2.938.769, 31 May 1960.

12. PORIEL, L., Pellet-ROStAing, S., LAMOTTE, V., LeMAIRE, M. Zirconium and Hafnium separation, part 2. Solid/liquid extraction in Hydrochloric acid aqueous solution with anion exchange resins. Separation Science and Technology., v. 41, p. 2711-2722, 2006.

13. SMOLIK, M., JAKÓBIK-KOLON, A., PORANSKI, M. Separation of zirconium and hafnium using Diphonix ${ }^{\circledR}$ chelating ion-exchange resin. Hydrometallurgy, v. 95, p. 350-353, 2009.

14. XU, Z., WU, Y., ZHANG, J., ZHANG, LI., WANG, LI. Equilibrium and Kinetic data of adsorption and separation for zirconium and hafnium onto MIBK extraction resin. Transactions of Nonferrous Metals Society of China., China, v. 20, p. 1527-1533, 2010. 